\title{
INVERSE PROBLEMS FOR DIFFERENTIAL EQUATIONS ON THE HALF-LINE HAVING A SINGULARITY IN AN INTERIOR POINT
}

\author{
A. FEDOSEEV
}

\begin{abstract}
Arbitrary order ordinary differential equations on the half-line having a nonintegrable singularity inside are studied under additional matching conditions for solutions at the singular point. We construct special fundamental systems of solutions for this class of differential equations, study their asymptotical, analytical and structural properties and the behavior of the corresponding Stokes multipliers. These fundamental systems of solutions are used in spectral analysis of differential operators with singularities. We study the inverse problem of recovering differential equation from the given WeylYurko matrix and prove the corresponding uniqueness theorem.
\end{abstract}

\section{Introduction}

Let us consider the differential equation

$$
\ell y(x):=y^{(n)}(x)+\sum_{j=0}^{n-2}\left(\frac{v_{j}}{(x-a)^{n-j}}+q_{j}(x)\right) y^{(j)}(x)=\lambda y(x), x>0
$$

with a non-integrable singularity in an interior point $0<a<\infty$. Here $q_{j}(x)$ are complexvalued functions, and $v_{j}$ are complex numbers. Let $\mu_{1}, \ldots, \mu_{n}$ be the roots of the characteristic polynomial

$$
\Delta(\mu)=\sum_{j=0}^{n} v_{j} \prod_{k=0}^{j-1}(\mu-k), \quad v_{n}=1, v_{n-1}=0 .
$$

Using the Viète's formulas one gets $\mu_{1}+\ldots+\mu_{n}=n(n-1) / 2$. For definiteness, let $n=2 m$, $\mu_{k}-\mu_{j} \neq s n, s \in \mathbb{Z} ; \Re \mu_{1}<\ldots<\Re \mu_{n}, \mu_{k} \neq 0,1,2, \ldots, n-3$ (the other cases require minor modifications). Denote $\theta=\Re\left(\mu_{n}-\mu_{1}\right), \theta_{j}=n-1-\theta-j$. We shall assume that $q_{j}(x)|x-a|^{\theta_{j}} \in L(0, T)$ and $q_{j}(x) \in L(T, \infty)$ for $j=\overline{0, n-2}$, and some $T>a$.

The paper deals with the differential equation (1) under additional matching conditions at the singular point $x=a$. We consider in some sense general matching conditions defined by a transition matrix

$$
A=\left[a_{k j}\right]_{k, j=\overline{1, n}}
$$

2000 Mathematics Subject Classification. 34E10, 34L05, 47E05, 34A55.

Key words and phrases. Ordinary differential equations, non-integrable singularity, fundamental systems of solutions, inverse problems. 
which connects solutions of equation (1) near the singular point. Differential equations with singularities inside the interval appear in different branches of mathematics and in applications. A wide class of differential equations with turning points inside the interval can be reduced to (1). Such problems produce different matching conditions defined by different matrices $A$. So it is actual to study differential equations with arbitrary defined matching conditions. In this paper we construct special fundamental systems of solutions for the differential equation (1) and study their asymptotical, analytic and structural properties. These fundamental systems of solutions are used in spectral analysis of differential operators with singularities inside the interval. In particular, we introduce and study the Weyl-Yurko solutions and the Weyl-Yurko matrix for equation (1) and prove the uniqueness theorem of recovering the operator $\ell$ from the given Weyl-Yurko matrix. For this purpose we use and develop ideas from [1].

We note that for higher-order differential equations with integrable coefficients, direct and inverse problems of spectral analysis and corresponding fundamental systems of solutions have been studied fairly completely (see [2]-[5] and the references therein). Uniqueness theorem for the equation (1) on the compact interval was proved in [6]. Some aspects of the spectral theory for equation (1) in the case of $n=2$ were investigated in [7]-[8].

Let $\lambda=\rho^{n}$. Take numbers $c_{j 0}, j=\overline{1, n}$ from the condition

$$
\prod_{j=1}^{n} c_{j 0}=\left(\operatorname{det}\left[\mu_{j}^{v-1}\right]_{j, v=\overline{1, n}}\right)^{-1},
$$

and consider the functions $C_{j}(x, \lambda)=(x-a)^{\mu_{j}} \widehat{C}_{j}(x, \lambda), j=\overline{1, n}$, where

$$
\widehat{C}_{j}(x, \lambda)=\sum_{k=0}^{\infty} c_{j k}(\rho(x-a))^{n k}, \quad c_{j k}=c_{j 0}\left(\prod_{s=1}^{k} \Delta\left(\mu_{j}+s n\right)\right)^{-1} .
$$

Here and in the sequel, $z^{\mu}=e^{\mu(\ln |z|+i \arg z)}, \arg z \in(-\pi, \pi]$. For each fixed $x$, the functions $C_{j}^{(v)}(x, \lambda)$ are entire in $\lambda$ of order $1 / n$. For $x>a$ and $x<a$ the functions $C_{j}(x, \lambda)$ satisfy the equation

$$
\ell_{0} y(x):=y^{(n)}(x)+\sum_{j=0}^{n-2} \frac{v_{j}}{(x-a)^{n-j}} y^{(j)}(x)=\lambda y(x),
$$

and for $|\rho(x-a)| \leq 1$,

$$
\left|\widehat{C}_{j}(x, \lambda)\right| \leq C, \quad\left|\widehat{C}_{j}^{(v)}(x, \lambda)\right| \leq C|\rho|^{n}|x-a|^{n-v}, \quad v=\overline{1, n-1} .
$$

Here and below, one and the same symbol $C$ denotes various positive constants in estimates. In view of (2) and Liouville's formula for the Wronskian, we have

$$
\operatorname{det}\left[C_{j}^{(v-1)}(x, \lambda)\right]_{j, v=\overline{1, n}} \equiv 1 .
$$


Denote

$$
C_{j}^{*}(x, \lambda)=\operatorname{det}\left[C_{k}^{(v)}(x, \lambda)\right]_{v=0, n-2}^{k=\overline{1, n} \backslash n-j+1}, \quad g(x, t, \lambda)=\sum_{j=1}^{n}(-1)^{n-j} C_{j}(x, \lambda) C_{n-j+1}^{*}(t, \lambda),
$$

where $k=\overline{1, n} \backslash n-j+1$ means $k=1, \ldots, n-j, n-j+2, \ldots, n$. The function $g(x, t, \lambda)$ is Green's function for the Cauchy problem $\ell_{0} y-\lambda y=f(x), y^{(v)}(a)=0, v=\overline{0, n-1}$. It follows from (3) that for $|\rho(x-a)| \leq 1, v=\overline{0, n-1}$,

$$
\left|C_{j}^{(v)}(x, \lambda)\right| \leq C\left|(x-a)^{\mu_{j}-v}\right|, \quad\left|C_{n-j+1}^{*}(x, \lambda)\right| \leq C\left|(x-a)^{n-1-\mu_{j}}\right| .
$$

Now we construct the functions $s_{j}(x, \lambda), j=\overline{1, n}$ from the following system of integral equations for $x>a$ and $x<a$ :

$$
s_{j}^{(v)}(x, \lambda)=C_{j}^{(v)}(x, \lambda)-\int_{a}^{x} \frac{\partial^{v}}{\partial x^{v}} g(x, t, \lambda)\left(\sum_{p=0}^{n-2} q_{p}(t) s_{j}^{(p)}(t, \lambda)\right) d t, v=\overline{0, n-1} .
$$

By (3) and (5), system (6) has a unique solution, and $s_{j}(x, \lambda)=(x-a)^{\mu_{j}} \hat{s}_{j}(x, \lambda)$, where

$$
\begin{aligned}
\left|\hat{s}_{j}^{(v)}(x, \lambda)-\widehat{C}_{j}^{(v)}(x, \lambda)\right| & \leq C|x-a|^{\theta-v} \varepsilon(x), \quad|\rho(x-a)| \leq 1, v=\overline{0, n-1}, \\
\varepsilon(x) & :=\sum_{s=0}^{n-2}\left|\int_{a}^{x}(t-a)^{\theta_{s}} q_{s}(t) d t\right| .
\end{aligned}
$$

In particular, this yields for $|\rho(x-a)| \leq 1$,

$$
\begin{aligned}
& \left|\hat{s}_{j}(x, \lambda)\right| \leq C,\left|\hat{s}_{j}^{(v)}(x, \lambda)\right| \leq C\left(|\rho|^{n}|x-a|^{n-v}+|x-a|^{\theta-v} \varepsilon(x)\right), v=\overline{1, n-1}, \\
& \left|s_{j}^{(v)}(x, \lambda)\right| \leq C\left|(x-a)^{\mu_{j}-v}\right|, v=\overline{0, n-1} .
\end{aligned}
$$

For each fixed $x$, the functions $s_{j}^{(v)}(x, \lambda)$ are entire in $\lambda$ of order $1 / n$. For $x>a$ and $x<a$ the functions $s_{j}(x, \lambda)$ satisfy equation (1). Taking (4) into account we get

$$
\operatorname{det}\left[s_{j}^{(v-1)}(x, \lambda)\right]_{j, v=\overline{1, n}} \equiv 1,
$$

and consequently, the functions $s_{j}(x, \lambda), j=\overline{1, n}$, form a fundamental system of solutions of equation (1). We will call $s_{j}(x, \lambda)$ the Bessel-type solutions for equation (1).

Let $s_{j,-}(x, \lambda), x>a$, be the Bessel-type solutions for the equation

$$
y_{-}^{(n)}(x)+\sum_{j=0}^{n-2}\left(\frac{v_{j}}{(x-a)^{n-j}}+(-1)^{j} q_{j}(2 a-x)\right) y_{-}^{(j)}(x)=\lambda y_{-}(x) .
$$

Then the functions $s_{j}^{R}(x, \lambda):=s_{j,-}(2 a-x, \lambda), x<a$, are solutions of equation (1). Clearly,

$$
s_{j}^{R}(x, \lambda)=e^{-i \pi \mu_{j}} s_{j}(x, \lambda), x<a .
$$


Denote $S_{k_{0}}=\left\{\rho: \arg \rho \in\left(\frac{k_{0} \pi}{n}, \frac{\left(k_{0}+1\right) \pi}{n}\right)\right\}$. In each sector $S_{k_{0}}$ the roots $R_{k}, k=\overline{1, n}$, of the equation $R^{n}-1=0$ can be numbered in such a way that

$$
\Re\left(\rho R_{1}\right)<\Re\left(\rho R_{2}\right)<\ldots<\Re\left(\rho R_{n}\right), \rho \in S_{k_{0}} .
$$

Clearly, $R_{k}=e^{\frac{i \pi \omega_{k}}{n}}$, where $\omega_{k}$ is a permutation of the numbers $0,1, \ldots, n-1$. In the sequel, $R_{k}^{\mu}:=e^{\frac{i \pi \mu \omega_{k}}{n}}$. It was shown in [1] that in each sector $S_{k_{0}}$, there exists a fundamental system of solutions $\left\{y_{k}(x, \rho)\right\}_{k=\overline{1, n}}, x>a, \rho \in \overline{S_{k_{0}}}$ of the differential equation (1) having the following properties.

1. For each $x>a$ and sufficiently large $\rho_{*}>0$, the functions $y_{k}^{(v)}(x, \rho), v=\overline{0, n-1}$, are analytic with respect to $\rho$ for $\rho \in S_{k_{0}},|\rho| \geq \rho_{*}$, are continuous for $\rho \in \overline{S_{k_{0}}},|\rho| \geq \rho_{*}$, and

$$
\left|y_{k}^{(v)}(x, \rho)\left(\rho R_{k}\right)^{-v} e^{-\rho R_{k}(x-a)}-1\right| \leq C\left(|\rho(x-a)|^{-1}+|\rho|^{-\delta_{0}}\right),
$$

for $x>a, \rho \in \overline{S_{k_{0}}},|\rho(x-a)| \geq 1$, where $\delta_{0}:=\min (1, \theta)$.

2. As $|\rho| \rightarrow \infty$,

$$
\operatorname{det}\left[y_{k}^{(v-1)}(x, \rho)\right]_{k, v=\overline{1, n}}=\rho^{m(n-1)} \Omega\left(1+O\left(\rho^{-\delta_{0}}\right)\right), x>a
$$

where $\Omega:=\operatorname{det}\left[R_{k}^{v-1}\right]_{k, v=\overline{1, n}} \neq 0$.

3. The relation

$$
y_{k}(x, \rho)=\sum_{j=1}^{n} b_{k j}^{+}(\rho) s_{j}(x, \lambda), x>a
$$

holds, where

$$
b_{k j}^{+}(\rho)=\beta_{j}^{0} R_{k}^{\mu_{j}} \rho^{\mu_{j}}\left(1+O\left(\rho^{-\delta_{0}}\right)\right),|\rho| \rightarrow \infty
$$

and

$$
\prod_{j=1}^{n} \beta_{j}^{0}=\left(\operatorname{det}\left[R_{k}^{\mu_{j}}\right]_{k, j=\overline{1, n}}\right)^{-1} \Omega .
$$

We note that the most important and non-trivial fact here is the asymptotical formula (14) for the Stokes multipliers $b_{k j}^{+}(\rho)$. We will call $y_{k}(x, \rho)$ the Birkhoff-type solutions for equation (1).

Let $y_{k,-}(x, \rho), x>a$, be the Birkhoff-type solutions for (9). Then the following functions $y_{k}(x, \rho):=y_{k,-}(2 a-x, \rho), x<a$, are solutions of (1). Symmetrically to (13), we have

$$
y_{k}(x, \rho)=\sum_{j=1}^{n} b_{k j}^{-}(\rho) s_{j}^{R}(x, \lambda), x<a,
$$

where

$$
b_{k j}^{-}(\rho)=\beta_{j}^{0} R_{k}^{\mu_{j}} \rho^{\mu_{j}}\left(1+O\left(\rho^{-\delta_{0}}\right)\right),|\rho| \rightarrow \infty,
$$


with the same constants $\beta_{j}^{0}$ as in (14). Since $\mu_{1}+\ldots+\mu_{n}=n(n-1) / 2$, it follows from (14), (15) and (17) that

$$
\operatorname{det}\left[b_{k j}^{ \pm}(\rho)\right]_{k, j=\overline{1, n}}=\rho^{m(n-1)} \Omega\left(1+O\left(\rho^{-\delta_{0}}\right)\right),|\rho| \rightarrow \infty .
$$

By virtue of (10) and (16),

$$
y_{k}(x, \rho)=\sum_{j=1}^{n} B_{k j}^{-}(\rho) s_{j}(x, \lambda), x<a
$$

where

$$
B_{k j}^{-}(\rho)=b_{k j}^{-}(\rho) e^{-i \pi \mu_{j}}
$$

It follows from (13) and (19) that

$$
\begin{aligned}
& s_{j}(x, \lambda)=\sum_{k=1}^{n} d_{j k}^{+}(\rho) y_{k}(x, \rho), x>a, \\
& s_{j}(x, \lambda)=\sum_{k=1}^{n} D_{j k}^{-}(\rho) y_{k}(x, \rho), x<a,
\end{aligned}
$$

where

$$
D_{j k}^{-}(\rho)=d_{j k}^{-}(\rho) e^{i \pi \mu_{j}} .
$$

and $\left[d_{j k}^{ \pm}(\rho)\right]_{j, k=\overline{1, n}}=\left(\left[b_{k j}^{ \pm}(\rho)\right]_{k, j=\overline{1, n}}\right)^{-1}$. Using (14) and (17) we infer

$$
d_{j k}^{ \pm}(\rho)=\left(\beta_{j}^{0}\right)^{-1} \rho^{-\mu_{j}}\left(d_{j k}+O\left(\rho^{-\delta_{0}}\right)\right),|\rho| \rightarrow \infty,
$$

where $\left[d_{j k}\right]_{j, k=\overline{1, n}}=\left(\left[R_{k}^{\mu_{j}}\right]_{k, j=\overline{1, n}}\right)^{-1}$. We rewrite (11) as follows

$$
y_{k}^{(v)}(x, \rho)=\left(\rho R_{k}\right)^{v} e^{\rho R_{k}(x-a)}[1]_{a}, x>a, \rho \in \overline{S_{k_{0}}},|\rho(x-a)| \geq 1,
$$

where $[1]_{a}=1+O\left(|\rho(x-a)|^{-1}+|\rho|^{-\delta_{0}}\right)$ for $|\rho(x-a)| \geq 1,|\rho| \rightarrow \infty$ uniformly in $x$ (i.e. $f(x, \rho)=$ $[1]_{a}$ means $|f(x, \rho)-1| \leq C\left(|\rho(x-a)|^{-1}+|\rho|^{-\delta_{0}}\right)$ for $\left.|\rho(x-a)| \geq 1\right)$. Similarly,

$$
y_{k}^{(v)}(x, \rho)=\left(-\rho R_{k}\right)^{v} e^{\rho R_{k}(a-x)}[1]_{a}, x<a, \rho \in \overline{S_{k_{0}}},|\rho(x-a)| \geq 1,
$$

In particular, (26) yields

$$
\operatorname{det}\left[y_{k}^{(v-1)}(x, \rho)\right]_{k, v=\overline{1, n}}=(-1)^{m} \rho^{m(n-1)} \Omega\left(1+O\left(\rho^{-\delta_{0}}\right)\right), x<a .
$$

Let a matrix $A=\left[a_{k j}\right]_{k, j=\overline{1, n}}$, $\operatorname{det} A \neq 0$, be given, where $a_{k j}$ are complex numbers. We introduce the functions $\left\{\sigma_{j}(x, \lambda)\right\}_{j=\overline{1, n}}, x \in J_{ \pm}$, where $J_{ \pm}:=\{x: \pm(x-a)>0\}$, by the formula

$$
\sigma_{j}(x, \lambda)= \begin{cases}s_{j}(x, \lambda), & x \in J_{-}, \\ \sum_{k=1}^{n} a_{k j} s_{k}(x, \lambda), & x \in J_{+} .\end{cases}
$$


For each fixed $x \neq a$, the functions $\sigma_{j}^{(v)}(x, \lambda), v=\overline{0, n-1}$, are entire in $\lambda$ of order $1 / n$. For $x \in J_{-}$ and $x \in J_{+}$, the functions $\sigma_{j}(x, \lambda)$ satisfy the differential equation (1), and according to (8),

$$
\operatorname{det}\left[\sigma_{j}^{(v-1)}(x, \lambda)\right]_{j, v=\overline{1, n}} \equiv \begin{cases}1, & x \in J_{-}, \\ \operatorname{det} A, & x \in J_{+} .\end{cases}
$$

The fundamental system of solutions $\left\{\sigma_{j}(x, \lambda)\right\}$ will be used for matching together solutions at the singular point $x=a$. More precisely, we shall say that a solution $y(x, \lambda)$ of equation (1) satisfies the matching condition generated by the transition matrix $A$, if $y(x, \lambda)$ can be represented in the form

$$
y(x, \lambda)=\sum_{j=1}^{n} \chi_{j}(\lambda) \sigma_{j}(x, \lambda) \text { for all } x \in J_{-} \cup J_{+},
$$

where the coefficients $\chi_{j}(\lambda)$ do not depend on $x$.

Using (13), (19), (21), (22) and (28) we get

$$
y_{k}(x, \rho)=\sum_{j=1}^{n} B_{k j}^{ \pm}(\rho) \sigma_{j}(x, \lambda), \quad \sigma_{j}(x, \lambda)=\sum_{k=1}^{n} D_{j k}^{ \pm}(\rho) y_{k}(x, \rho) \quad x \in J_{ \pm},
$$

where $B_{k j}^{-}(\rho), D_{j k}^{-}(\rho)$ are defined by (20) and (23),

$$
D_{j k}^{+}(\rho)=\sum_{s=1}^{n} a_{s j} d_{s k}^{+}(\rho)
$$

and $B_{k j}^{+}(\rho)$ can be found from the linear system

$$
b_{k l}^{+}(\rho)=\sum_{j=1}^{n} B_{k j}^{+}(\rho) a_{l j}
$$

i.e. $\left[B_{k j}^{+}(\rho)\right]_{k, j=\overline{1, n}}=\left[b_{k j}^{+}(\rho)\right]_{k, j=\overline{1, n}}\left(A^{T}\right)^{-1}$, where $T$ denotes the transposition. Clearly,

$$
\begin{gathered}
{\left[D_{j k}^{ \pm}(\rho)\right]_{j, k=\overline{1, n}}=\left(\left[B_{k j}^{ \pm}(\rho)\right]_{k, j=\overline{1, n}}\right)^{-1},} \\
\operatorname{det}\left[B_{k j}^{-}(\rho)\right]_{k, j=\overline{1, n}}=(-1)^{m} \operatorname{det}\left[b_{k j}^{-}(\rho)\right]_{k, j=\overline{1, n}}, \operatorname{det}\left[B_{k j}^{+}(\rho)\right]_{k, j=\overline{1, n}}=\frac{\operatorname{det}\left[b_{k j}^{+}(\rho)\right]_{k, j=\overline{1, n}}}{\operatorname{det} A} .
\end{gathered}
$$

For definiteness, in the sequel we confine ourselves to the most important particular case when $a_{k j}=0$ for $k<j$. Let $\varphi_{j}(x, \lambda), j=\overline{1, n}$, be solutions of equation (1) satisfying the initial conditions

$$
\varphi_{j}^{(v-1)}(0, \lambda)=\delta_{j v}, j, v=\overline{1, n}
$$

( $\delta_{j v}$ is the Kronecker delta) and also the matching condition generated by the transition matrix $A$. 
Theorem 1. ( $\left.i_{1}\right)$ For each fixed $x \neq a$, the functions $\varphi_{j}^{(v)}(x, \lambda), v=\overline{0, n-1}$, are entire in $\lambda$ of order $1 / n$, and

$$
\operatorname{det}\left[\varphi_{j}^{(v-1)}(x, \lambda)\right]_{j, v=\overline{1, n}} \equiv \begin{cases}1, & x \in J_{-}, \\ \operatorname{det} A, & x \in J_{+} .\end{cases}
$$

(i $\left.i_{2}\right)$ For $|\rho(x-a)| \geq 1, \rho \in \overline{S_{k_{0}}}, v=\overline{0, n-1}, j=\overline{1, n},|\rho| \rightarrow \infty$, the following asymptotical formulas are valid

$$
\begin{gathered}
\varphi_{j}^{(v)}(x, \lambda)=\frac{1}{n} \sum_{k=1}^{n}\left(-\rho R_{k}\right)^{v+1-j} e^{-\rho R_{k} x}[1]_{a}, x \in J_{-}, \\
\varphi_{j}^{(v)}(x, \lambda)=\frac{1}{n} \sum_{l, k=1}^{n}\left(-\rho R_{k}\right)^{1-j}\left(\rho R_{l}\right)^{v}\left(\xi_{k l}^{0}+O\left(\rho^{-\delta_{1}}\right)\right) e^{-\rho R_{k} a} e^{\rho R_{l}(x-a)}[1]_{a}, x \in J_{+}
\end{gathered}
$$

where $\delta_{1}:=\min \left(1, \min _{l} \Re\left(\mu_{l+1}-\mu_{l}\right)\right)$, and

$$
\xi_{k j}^{0}=\sum_{s=1}^{n} a_{s s} R_{k}^{\mu_{s}} d_{s j} e^{-i \pi \mu_{s}}
$$

Proof. Since the functions $\varphi_{j}(x, \lambda)$ satisfy the matching conditions, the following representation holds

$$
\varphi_{j}(x, \lambda)=\sum_{s=1}^{n} \chi_{j s}(\lambda) \sigma_{s}(x, \lambda), x \in J_{-} \cup J_{+} .
$$

According to the initial conditions (34), the coefficients $\chi_{j s}(\lambda)$ can be found from the linear systems

$$
\sum_{s=1}^{n} \chi_{j s}(\lambda) \sigma_{s}^{(v-1)}(0, \lambda)=\delta_{j v}, v=\overline{1, n},
$$

for each $j=\overline{1, n}$. In view of (29), the determinant of (39) is equal to 1. Solving (39) by Cramer's rule we obtain that the functions $\chi_{j s}(\lambda)$ are entire in $\lambda$ of order $1 / n$, and $\operatorname{det}\left[\chi_{j s}(\lambda)\right]_{j, s=\overline{1, n}} \equiv 1$. Together with (38) and (29) this yields $\left(i_{1}\right)$.

Applying the fundamental system of solutions $\left\{y_{k}(x, \rho)\right\}_{k=\overline{1, n}}$ we expand $\varphi_{j}(x, \lambda)$ for $x \in$ $J_{+}$and $x \in J_{-}$separately:

$$
\varphi_{j}(x, \lambda)=\sum_{k=1}^{n} A_{j k}^{ \pm}(\rho) y_{k}(x, \lambda), x \in J_{ \pm} .
$$

Using the initial conditions (34) we calculate

$$
\sum_{k=1}^{n} A_{j k}^{-}(\rho) y_{k}^{(v-1)}(0, \rho)=\delta_{j v}, j, v=\overline{1, n},
$$

Hence, by virtue of (26) and (27),

$$
A_{j k}^{-}(\rho)=(-\rho)^{1-j} e^{-\rho R_{k} a}\left(\alpha_{j k}+O\left(\rho^{-\delta_{0}}\right)\right),
$$


where the numbers $\alpha_{j k}$ can be found from the linear systems

$$
\sum_{k=1}^{n} \alpha_{j k} R_{k}^{v-1}=\delta_{j v}, v=\overline{1, n}
$$

for each $j=\overline{1, n}$. It is easy to verify that $\alpha_{j k}=R_{k}^{1-j} / n$, and consequently,

$$
A_{j k}^{-}(\rho)=\frac{1}{n}\left(-\rho R_{k}\right)^{1-j} e^{-\rho R_{k} a}\left(1+O\left(\rho^{-\delta_{0}}\right)\right) .
$$

Substituting (41) and (26) into (40), we arrive at (36).

In order to calculate $A_{j k}^{+}(\rho)$ we use the matching condition generated by the transition matrix A. Substituting (30) into (40) we get

$$
\varphi_{j}(x, \lambda)=\sum_{s=1}^{n} \sigma_{s}(x, \lambda) \sum_{k=1}^{n} A_{j k}^{ \pm}(\rho) B_{k s}^{ \pm}(\rho), x \in J_{ \pm} .
$$

Taking (38) into account, we infer

$$
\sum_{k=1}^{n} A_{j k}^{+}(\rho) B_{k s}^{+}(\rho)=\sum_{k=1}^{n} A_{j k}^{-}(\rho) B_{k s}^{-}(\rho) .
$$

According to (32), the last equality yields

$$
A_{j k}^{ \pm}(\rho)=\sum_{s=1}^{n} A_{j s}^{\mp}(\rho) \xi_{s k}^{ \pm}(\rho)
$$

where

$$
\xi_{s k}^{ \pm}(\rho)=\sum_{l=1}^{n} B_{s l}^{\mp}(\rho) D_{l k}^{ \pm}(\rho) .
$$

Thus, $\left[\xi_{k j}^{ \pm}(\rho)\right]_{k, j=\overline{1, n}}=\left[B_{k j}^{\mp}(\rho)\right]_{k, j=\overline{1, n}}\left[D_{j k}^{ \pm}(\rho)\right]_{j, k=\overline{1, n}}$, and

$$
\left[\xi_{k j}^{-}(\rho)\right]_{k, j=\overline{1, n}}=\left(\left[\xi_{k j}^{+}(\rho)\right]_{k, j=\overline{1, n}}\right)^{-1} .
$$

Together with (32) and (33) this yields

$$
\operatorname{det}\left[\xi_{k j}^{+}(\rho)\right]_{k, j=\overline{1, n}}=(-1)^{m} \operatorname{det} A \operatorname{det}\left[b_{k j}^{-}(\rho)\right]_{k, j=\overline{1, n}}\left(\operatorname{det}\left[b_{k j}^{+}(\rho)\right]_{k, j=\overline{1, n}}\right)^{-1},
$$

and, in view of (18)

$$
\operatorname{det}\left[\xi_{k j}^{+}(\rho)\right]_{k, j=1, n}=(-1)^{m} \operatorname{det} A\left(1+O\left(\rho^{-\delta_{0}}\right)\right),|\rho| \rightarrow \infty .
$$

By virtue of (17) and (20),

$$
B_{k j}^{-}(\rho)=\beta_{j}^{0} R_{k}^{\mu_{j}} e^{-i \pi \mu_{j}} \rho^{\mu_{j}}\left(1+O\left(\rho^{\delta_{0}}\right)\right),|\rho| \rightarrow \infty .
$$


It follows from (24) and (31) that

$$
D_{j k}^{+}(\rho)=\left(\beta_{j}^{0}\right)^{-1} a_{j j} \rho^{-\mu_{j}}\left(d_{j k}+O\left(\rho^{-\delta_{0}}\right)+O\left(\rho^{-\Re\left(\mu_{j+1}-\mu_{j}\right)}\right)\right),|\rho| \rightarrow \infty .
$$

Substituting (45) and (46) into (43) we calculate

$$
\xi_{s k}^{+}=\xi_{s k}^{0}+O\left(\rho^{-\delta_{1}}\right), \quad|\rho| \rightarrow \infty,
$$

where the numbers $\xi_{s k}^{0}$ are defined by (37). Moreover, (44) and (47) imply

$$
\operatorname{det}\left[\xi_{k j}^{0}\right]_{k, j=\overline{1, n}}=(-1)^{m} \operatorname{det} A .
$$

Using (41), (42) and (47) we deduce

$$
A_{j k}^{+}(\rho)=\frac{1}{n} \sum_{s=1}^{n}\left(-\rho R_{s}\right)^{1-j} e^{-\rho R_{s} a}\left(\xi_{s k}^{0}+O\left(\rho^{-\delta_{1}}\right)\right),|\rho| \rightarrow \infty,
$$

hence (40) and (25) yield (1). Theorem 1 is proved.

Denote

$$
\xi_{s}^{0}=\operatorname{det}\left[\xi_{k j}^{0}\right]_{k=\overline{1, s}}^{j=\overline{n-s+1, n}}, s=\overline{1, n} .
$$

In particular, according to (48), $\xi_{n}^{0}=(-1)^{m} \operatorname{det} A \neq 0$.

We shall assume that

$$
\xi_{s}^{0} \neq 0, s=\overline{1, n-1} .
$$

Condition (50) is called the regularity condition for matching.

We introduce the functions

$$
\begin{gathered}
\Delta_{j}(\rho)=\operatorname{det}\left[y_{k}(0, \rho), \ldots, y_{k}^{(j-1)}(0, \rho), \xi_{k j+1}^{+}(\rho), \ldots, \xi_{k n}^{+}(\rho)\right]_{k=\overline{1, n}}^{T}, j=\overline{1, n-1}, \\
\Delta_{n}(\rho)=\operatorname{det}\left[y_{k}^{(v-1)}(0, \rho)\right]_{k, v=\overline{1, n}} .
\end{gathered}
$$

It is clear that

$$
\left.\Delta_{j}(\rho)=\sum_{1 \leq s_{1}<\ldots<s_{n} \leq n} \operatorname{det}\left[\xi_{s_{p} k}^{+}\right]_{k=\overline{j+1, n} ; p=\overline{1, n-j}} \operatorname{det}\left[y_{s_{p}}^{(v-1)}(0, \rho)\right)\right]_{p, v=\overline{1, j}} .
$$

Substituting (26) and (47) into (52) we get for $|\rho| \rightarrow \infty, \rho \in S_{k_{0}}, j=\overline{1, n-1}$ :

$$
\Delta_{j}(\rho)=(-\rho)^{1+\ldots+(j-1)} e^{\rho\left(R_{n}+\ldots+R_{n-j+1}\right) a}\left(\Delta_{j}^{0}(\rho)+O\left(\rho^{-\delta_{1}}\right)\right)
$$

where

$$
\begin{aligned}
\Delta_{j}^{0}(\rho) & =\Delta_{j, 0}+\Delta_{j, 1} e^{\rho\left(R_{n-j}-R_{n-j+1}\right) a}, \\
\Delta_{j, 0} & =d_{0} \operatorname{det}\left[\xi_{s k}^{0}\right]_{s=\overline{1, n-j} ; k=\overline{j+1, n}}, d_{0} \neq 0, \\
\Delta_{j, 1} & =d_{1} \operatorname{det}\left[\xi_{s k}^{0}\right]_{s=\overline{1, n-j-1}, n-j+1 ; k=\overline{j+1, n},}
\end{aligned}
$$

$d_{0}$ and $d_{1}$ some constants that do not depend on $\rho$. By the regularity condition for matching, we have $\Delta_{j, 0} \neq 0$. Using (53), by the well-known methods (see, for example, [9]), one can obtain the following assertion. 
Theorem 2. Let $\left\{\rho_{l j}\right\}$ be the zeros of $\Delta_{j}(\rho)$. Denote $G_{\delta, j}:=\left\{\rho:\left|\rho-\rho_{l j}\right| \geq \delta\right\}$. Then

$$
\left|\Delta_{j}(\rho)\right| \geq C_{\delta}\left|\rho^{j(j-1) / 2} e^{\rho\left(R_{n}+\ldots+R_{n-j+1}\right) a}\right|, \rho \in \overline{S_{k_{0}}} \cup G_{\delta, j},|\rho| \rightarrow \infty
$$

We now introduce the functions $\Phi_{j}(x, \lambda), j=\overline{1, n}$, which are solutions of equation (1) with the conditions

$$
\Phi_{j}^{(v-1)}(0, \lambda)=\delta_{v j}, v=\overline{1, j}, \quad \Phi_{j}(x, \lambda)=O\left(e^{\rho R_{j} x}\right), x \rightarrow \infty, j=\overline{1, n}, \rho \in S_{k_{0}},
$$

and with the matching condition generated by the transition matrix $A$. We will call $\Phi_{j}(x, \lambda)$, $j=\overline{1, n}$, the Weyl-Yurko solution for the differential equation (1). Using (34) we calculate

$$
\Phi_{j}(x, \lambda)=\varphi_{j}(x, \lambda)+\sum_{k=j+1}^{n} M_{j k}(\lambda) \varphi_{k}(x, \lambda)
$$

where

$$
M_{j k}(\lambda)=\Phi_{j}^{(k-1)}(0, \lambda), 1 \leq j<k \leq n .
$$

It follows from (35) and (56) that

$$
\operatorname{det}\left[\Phi_{j}^{(v-1)}(x, \lambda)\right]_{j, v=1, n} \equiv \begin{cases}1, & x \in J_{-} \\ \operatorname{det} A, & x \in J_{+}\end{cases}
$$

Relation (56) can be written in the form

$$
\Phi(x, \lambda)=\varphi(x, \lambda) M^{T}(\lambda)
$$

where $\Phi(x, \lambda)=\left[\Phi_{j}^{(v-1)}(x, \lambda)\right]_{j, v=\overline{1, n}}, \quad \varphi(x, \lambda)=\left[\varphi_{j}^{(v-1)}(x, \lambda)\right]_{j, v=\overline{1, n}}, \quad M(\lambda)=\left[M_{j k}(\lambda)\right]_{j, k=\overline{1, n}}$, $M_{j k}(\lambda):=\delta_{j k}$ for $j \geq k$. We will call $M(\lambda)$ the Weyl-Yurko matrix for equation (1), since it is a generalization of the concept of the Weyl-Yurko matrix introduced in [4].

Theorem 3. For $|\rho(x-a)| \geq 1, \rho \in \overline{S_{k_{0}}} \cap G_{\delta, j},|\rho| \rightarrow \infty, j, v=\overline{1, n}$ the following estimates hold

$$
\begin{array}{ll}
\left|\Phi_{j}^{(v-1)}(x, \lambda)\right| \leq C\left|\rho^{v-j} e^{-\rho R_{n-j+1} x}\right|, & x \in J_{-}, \\
\left|\Phi_{j}^{(v-1)}(x, \lambda)\right| \leq C\left|\rho^{v-j} e^{\rho R_{j} x} e^{-\rho\left(R_{n-j+1}+R_{j}\right) a}\right|, & x \in J_{+} .
\end{array}
$$

Proof. Since the functions $\Phi_{j}(x, \lambda)$ satisfy the matching condition generated by the transition matrix $A$, the following representation holds

$$
\Phi_{j}(x, \lambda)=\sum_{s=1}^{n} \chi_{j s}^{0}(\lambda) \sigma_{s}(x, \lambda), \quad x \in J_{-} \cup J_{+}
$$


where the coefficients $\chi_{j s}^{0}(\lambda)$ do not depend on $x$. Applying the fundamental system of solutions $\left\{y_{k}(x, \rho)\right\}_{k=\overline{1, n}}$ we expand $\Phi_{j}(x, \lambda)$ for $x \in J_{-}$and $x \in J_{+}$separately:

$$
\Phi_{j}(x, \lambda)=\sum_{k=1}^{n} a_{j k}^{ \pm}(\rho) y_{k}(x, \rho), \quad x \in J_{ \pm} .
$$

Substituting (30) into (61) we get

$$
\Phi_{j}(x, \lambda)=\sum_{s=1}^{n} \sigma_{s}(x, \lambda) \sum_{k=1}^{n} a_{j k}^{ \pm}(\rho) B_{k s}^{ \pm}(\rho), \quad x \in J_{ \pm},
$$

and consenquently,

$$
\chi_{j s}^{0}(\lambda)=\sum_{k=1}^{n} a_{j k}^{+}(\rho) B_{k s}^{+}(\rho)=\sum_{k=1}^{n} a_{j k}^{-}(\rho) B_{k s}^{-}(\rho) .
$$

According to (32), this yields

$$
a_{j k}^{+}(\rho)=\sum_{s=1}^{n} a_{j s}^{-}(\rho) \xi_{s k}^{+}(\rho),
$$

where the functions $\xi_{s k}^{+}(\rho)$ are defined by (43). Using (55), (62) and (64) we get

$$
\left.\begin{array}{lr}
\sum_{k=1}^{n} a_{j k}^{-}(\rho) y_{k}^{(v-1)}(0, \rho)=\delta_{j v}, & v=\overline{1, j}, \\
\sum_{k=1}^{n} a_{j k}^{-}(\rho) \xi_{k v}^{+}(\rho)=a_{j v}^{+}(\rho) \equiv 0, & v=\overline{j+1, n}
\end{array}\right\}
$$

For each $j=\overline{1, n}$, (65) is a linear system with respect to $a_{j k}^{-}(\rho)$. The determinant of this system is $\Delta_{j}(\rho)$. Solving (65) by Cramer's rule and using (26), (27), (47), (54) and (64) we get for $\rho \in$ $\overline{S_{k_{0}}} \cap G_{\delta, j}, j=\overline{1, n-1}$ :

$$
\begin{array}{lr}
\left|a_{j k}^{-}(\rho)\right| \leq C\left|\rho^{1-j} e^{-\rho R_{n-j+1} a}\right|, & k=\overline{1, n-j+1}, \\
\left|a_{j k}^{-}(\rho)\right| \leq C\left|\rho^{1-j} e^{-\rho R_{k} a}\right|, & k=\overline{n-j+1, n}, \\
\left|a_{j k}^{+}(\rho)\right| \leq C\left|\rho^{1-j} e^{-\rho R_{n-j+1} a}\right|, & k=\overline{1, j},
\end{array}
$$

and $a_{j k}^{+}(\rho) \equiv 0, k=\overline{j+1, n}$. Substituting (66), (25) and (26) into (62) we arrive at (60). For $j=n$ estimates (60) obtained similarly. Theorem 3 is proved.

Now we consider the inverse problem of recovering $\ell$ from the given Weyl-Yurko matrix $M(\lambda)$. Let us prove the uniqueness theorem for the solution of the inverse problem. For this purpose we agree that together with $\ell$ we consider a differential equation $\tilde{\ell}$ of the same form but with different coefficients $\tilde{q}_{j}$ and $\tilde{v}_{j}$. If a certain symbol $\gamma$ denotes an object related to $\ell$, then the corresponding symbol $\tilde{\gamma}$ with tilde will denote the analogous object related to $\tilde{\ell}$.

Theorem 4. If $M(\lambda)=\widetilde{M}(\lambda)$, then $\ell=\tilde{\ell}$. Thus, the specification of the Weyl-Yurko matrix $M(\lambda)$ determines the differential equation (1) uniquely. 
Proof. Define the matrix $\left.\mathscr{P}(x, \lambda)=\left[\mathscr{P}_{j k}(x, \lambda)\right)\right]_{j, k=\overline{1, n}}$ by the following formula $\mathscr{P}(x, \lambda)=$ $\Phi(x, \lambda)(\widetilde{\Phi}(x, \lambda))^{-1}$ or, in view of (58), in the coordinates

$$
\begin{gathered}
\mathscr{P}_{j k}(x, \lambda)=(\eta(x))^{-1} \operatorname{det}\left[\widetilde{\Phi}_{s}(x, \lambda), \ldots, \widetilde{\Phi}_{s}^{(k-2)}(x, \lambda),\right. \\
\left.\Phi_{s}^{(j-1)}(x, \lambda), \widetilde{\Phi}_{s}^{(k)}(x, \lambda), \ldots, \widetilde{\Phi}_{s}^{(n-1)}(x, \lambda)\right]_{s=\overline{1, n}},
\end{gathered}
$$

where $\eta(x)=1$ for $x \in J_{-}$and $\eta(x)=\operatorname{det} A$ for $x \in J_{+}$. Denote $G_{\delta}=\bigcap_{j=1}^{n-1} G_{\delta, j}$. By virtue of (60) and (67), we have for each fixed $x \neq a$ :

$$
\mathscr{P}_{j k}(x, \lambda)=O\left(\rho^{j-k}\right),|\rho| \rightarrow \infty, \rho \in G_{\delta} .
$$

Using (59) and the assumption of Theorem 4 , we transform the matrix $\mathscr{P}(x, \lambda)$ as follows

$$
\mathscr{P}_{j k}(x, \lambda)=\varphi(x, \lambda)(\widetilde{\varphi}(x, \lambda))^{-1} .
$$

Taking (35) into account we conclude that for each fixed $x \neq a$, the functions $\mathscr{P}_{j k}(x, \lambda)$ are entire in $\lambda$. Using (68), the maximum modulus principle and Liouville's theorem, we get $\mathscr{P}_{1 k}(x, \lambda) \equiv 0$ for $k=\overline{2, n}$, and $\mathscr{P}_{11}(x, \lambda) \equiv \mathscr{P}(x)$ does not depend in $\lambda$. Therefore,

$$
\Phi_{j}(x, \lambda) \equiv \mathscr{P}(x) \widetilde{\Phi}_{j}(x, \lambda) .
$$

Using the asymptotical formulas (36) and (1) for the function $\varphi_{n}(x, \lambda) \equiv \Phi_{n}(x, \lambda)$, we get from

(69) that $\mathscr{P}(x, \lambda) \equiv 1$. Hence $\Phi_{j}(x, \lambda) \equiv \widetilde{\Phi}_{j}(x, \lambda)$ for all $x, \lambda$, $j$. This yields $\ell=\tilde{\ell}$, and Theorem 4 is proved.

\section{References}

[1] V. A. Yurko, Inverse problem for differential equations with a singularity, Differ. Uravneniya, 28 (1992), 13551362; English transl. in Differential Equations, 28 (1992), 1100-1107.

[2] R. Beals, P. Deift and C. Tomei, Direct and inverse scattering on the line, Math. Surveys and Monographs, v. 28, Amer. Math. Soc. Providence: RI, 1988.

[3] M. A. Naimark, Linear Differential Operators, 2nd ed., "Nauka“ Moscow 1969; English tranl. of 1st ed., Parts I,II, Ungar, New York 1967, 1968.

[4] V. A. Yurko, Inverse Spectral Problems for Differential Operators and their Applications, Gordon and Breach, New York, 2000.

[5] V. A. Yurko, Method of Spectral Mappings in the Inverse Problem Theory. Inverse and Ill-posed Problems Series. VSP. Utrecht, 2002.

[6] V. A. Yurko, Higher-order differential equations having a singularity in an interior point, Result. Math., 42 (2002), 177-191.

[7] V. A. Yurko, Integral transforms connected with differential operators having singularities inside the interval, Integral Transforms and Special Functions, 5 (1997), 309-322.

[8] V. A. Yurko, Inverse problems for differential equations with singularities lying inside the interval, Journal of Inverse and Ill-Posed Problems, 8, (2000), 89-103.

[9] R. Bellman and K. Cooke, Differential-difference Equations, Academic Press, New York, 1963.

Department of Mathematics, Saratov University, Astrakhanskaya 83, Saratov 410012, Russia, Russian Federation.

E-mail: fedoseev_ae@mail.ru 